\title{
Nonmetastatic Colon Cancer Model C26 Upregulates Glycolysis in Osteocytes in Vitro and Bone in Vivo
}

\author{
M. Roarke Tollar, ${ }^{1,3}$ Matt Prideaux, ${ }^{2}$ Fabrizio Pin, ${ }^{2}$ Lynda Bonewald ${ }^{2,3}$ \\ Indiana University School of Medicine $;^{1}$ Indiana University School of Medicine Department of \\ Anatomy, Cell Biology and Physiology; ${ }^{2}$ Indiana Center for Musculoskeletal Health; ${ }^{3}$ Indiana \\ University, Indianapolis, IN, USA. \\ Background: Developing effective treatments for musculoskeletal complications in cancer \\ patients requires understanding metabolic effects of cancer on bone, and particularly \\ osteocytes, the most abundant bone cell and key regulator of bone remodeling. However, little \\ is known regarding how cancer impacts normal osteocyte energy metabolic pathways, such as \\ glycolysis. Given that changes in metabolism are important regulators of cellular function, it is \\ essential to determine how osteocyte metabolism is disrupted by cancer and how this may \\ impact skeletal and whole-body health.
}

Methods: Mice inoculated with saline $(\mathrm{N}=5)$ or $\mathrm{C} 26$ cells $(\mathrm{N}=6)$ were sacrificed after 2 weeks. Bones were harvested for metabolic profiling by GC-MS, gene expression by RT-PCR and bone morphology by $\mu \mathrm{CT}$. Differentiated IDG-SW3 osteocyte-like cells were cocultured with C26 cells for 12-24hrs and metabolites and gene expression analyzed by GC-MS and RT-PCR.

Results: Trabecular bone mass was significantly decreased in the C26 mice. GC-MS analysis revealed decreased glucose in C26 mice tibiae, but no change in lactate. The bone resorption promoting gene Rankl was upregulated, whereas the inhibitor Opg was unchanged. Bone mineralization regulators Mepe and Phex were decreased. In vitro metabolic studies revealed increased glucose and lactate in IDG-SW3 cell lysate; culture media glucose levels were decreased whereas lactate was increased in the co-cultures with C26 cells. RT-PCR demonstrated increases in the glycolysis promoter Hif1 $\alpha$ in addition to glycolysis pathway genes including Glut1, Hk2, Slc16a3 and Pdk1. Rankl was also increased in the IDG-SW3 cells cocultured with the C26 cells whereas Opg, Phex, and Mepe were downregulated.

Conclusion: Glycolysis is upregulated in mouse bone and in vitro IDG-SW3 cells exposed to cancer. Our study provides novel understanding for how cancer affects bone metabolism. Integrating these results with whole body metabolism will aid in the development of novel therapeutic strategies to target musculoskeletal and systemic complications of cancer. 